\title{
PHOTOREACTIVATION ACTION SPECTRUM OF MOSS SPORES (BRYUM PSEUDO-TRIQUETRUM) INACTIVATED BY ULTRAVIOLET LIGHT
}

\author{
KENJIRO KINUGAWA AND ICHIRO OTSUKA* \\ Laboratory of Genetics and Plant Breeding, Faculty of Agriculture, \\ University of Osaka Prefecture, Sakai 591 \\ and \\ *Kihara Institute for Biological Research, Mishima 411
}

Received October 29, 1971

It has been well established that photoenzymatic splitting of pyrimidine dimers plays a principal role in the process of photoreactivation of u.v. effects on biological functions (Setlow 1966). Efficiency of enzymatic photoreactivation depends on temperature of the photoreactivating system and on intensity and energy of photoreactivating light (e. g., Jagger 1967). The action spectra of photoreactivation in microorganisms have been reported by many authors. They investigated in vivo photoreactivation with viral (Dulbecco 1950), bacterial (Jagger et al. 1969; Kondo and Kato 1966), algal (Van Baalen 1968) and fungal (Kelner 1951; Jagger et al. 1970) DNAs and in vitro photoreactivation with transforming DNA (Rupert 1960; Setlow and Boiling 1963), while Schiff et al. (1961) studied on Euglena in which photoreactivation probably acts on u.v. damage to chloroplast DNA. In most cases, effective wavelengths were found to lie in the visible to near-u.v. region (3000-4000 $)$, while a peak around $4300 \AA$ was found for Streptomyces griseus (Kelner 1951; Jagger et al. 1970) and Agmenellum quadruplicatum (Van Baalen 1968). Studies on photoreactivation in higher plants are difficult because of their multicellular structure. Ikenaga and Mabuchi (1966), Amano and Mabuchi (1966), Matsumura and Mabuchi (1966) and Fujii (1969) showed that maize plants possess photoreactivating ability. Saito and Werbin (1969) demonstrated existence of photoreactivating enzyme in the pinto bean plant.

The moss plant is at a rather higher evolutionary level. Nevertheless, it is easy to study photoreactivation of u.v. effects in the moss because of its unicelliate spore and hyphae-like protonema. This paper deals mainly with action spectrum of photoreactivation of u.v.-depressed germination of moss spores.

\section{MATERIAL AND METHODS}

The sporogons of moss (Bryum pseudo-triquetrum) were collected from the wild population on rocks along a small stream in Kiyotaki valley, Kyoto, Japan. It has been found that spores from a single sporogon are sufficiently uniform as the material for the present investigation.

Throughout the experiment, an artificial culture medium for spores was used. The 
medium consisted of $0.1 \mathrm{~g} \mathrm{Ca}\left(\mathrm{NO}_{3}\right)_{2} \cdot 4 \mathrm{H}_{2} \mathrm{O}, 0.01 \mathrm{~g} \mathrm{MgSO}_{4} \cdot 7 \mathrm{H}_{2} \mathrm{O}$ and $0.04 \mathrm{~g} \mathrm{~K}_{2} \mathrm{HPO}_{4}$ in 1000 $\mathrm{ml}$ deionized water and adjusted to $\mathrm{pH} 6.5$ by $1 \mathrm{~N} \mathrm{HCl}$ (Kinugawa 1966). The usual incubation was carried out at $25^{\circ} \mathrm{C}$ under 1000 to $1200 \mathrm{Lux}$ illumination from daylighttype fluorescent lamps. Irradiation with u.v. was performed using a $15 \mathrm{~W}$ germicidal lamp (GL 15, Toshiba Elec. Co.) emitting mainly $2537 \AA$. The u.v. dose administered was $3 \times 10^{4} \mathrm{ergs} / \mathrm{mm}^{2}$ with intensity $60 \mathrm{ergs} / \mathrm{mm}^{2} \cdot \mathrm{sec}$ at $20 \mathrm{~cm}$ from the lamp.

In the experiment for assaying a temperature effect on photoreactivation, the medium temperature was measured by a copper-constantan thermojunction. The medium within a small bottle was kept at a given temperature by steeping the bottle in a water bath. Temperature of the water bath was adjusted manually with hot or cool water. Spores collected from a single sporogon were floated in a monolayer over the medium surface and then irradiated with u.v. Immediately after u.v. irradiation, spores were transferred to a separate medium which was kept at $2^{\circ}$ or $25^{\circ} \mathrm{C}$, and illuminated $(1200$ Lux) by a daylight-type fluorescent lamp for one hour. The cultures were then placed in the dark at $25^{\circ} \mathrm{C}$ for 20 hours and followed by the usual incubation under light. Germinations were counted after seven day incubation with 500 spores in each culture.

The experiment for the action spectrum was repeated four times, and each replication consisted of the spores from a single sporogon. Spores collected were scattered on a thin glass plate and stored in a desiccator. The glass plate was steeped into culture medium in order to disperse the spores in a monolayer over the medium surface. Then the plate was kept in dark at $25^{\circ} \mathrm{C}$ for 10 to 60 minutes before u.v. irradiation. The imbibition interval before u.v. has been found to affect very little on the u.v. sensitivity of spores. Within 20 seconds after u.v. irradiation, photoreactivation treatment with monochromatic light commenced. To obtain photoreactivating light of different spectral bands from a high pressure marcury lamp (SHL 1000, Toshiba Elec. Co.) or a projector filament lamp (750 W, Toshiba Elec. Co.), the following filters were used: (1) Band pass glass filter UV-D1C (Toshiba Elec. Co.) transparent to $3000-4000 \AA$ with a peak at $3600 \AA$, and interference filters having a maximum transmission at the following wavelengths with band widths less than $200 \AA$ (Shonan Kogaku Co.) : (2) $4070 \AA$, (3) $4365 \AA$, (4) $4610 \AA$, (5) $4910 \AA$, (6) $5350 \AA$, (7) $5690 \AA$, (8) $5990 \AA$, and (9) $6450 \AA$. Filters (2), (3) and (5) were used for the marcury lamp, since their peak wavelengths fit to some of the emission lines of marcury arc. The rest of the filters were used in combination with the filament lamp. The equipment used for the irradiation of monochromatic light was constructed with a lamp, slit, filter, and a chamber for containing spore culture. Filters were placed far from the lamp position but near to the chamber. Intensity of photoreactivating light was measured by means of a pyrheliometer essentially consists of thermopiles covered with a transparent glass dome. Since the glass dome gives constant transparencies (ca. 93\%) to any wavelengths above $3600 \AA$, thermopiles produce voltages proportional to incident intensity of light and the proportionality constant is independent of wavelengths used in this experiment. Resulting voltages were measured by a DC microvolt meter (Toa Elec. Co.) and the intensities of photoreactivating light were regulated to be equal for all the wavelengths used by adjusting the distance from filter to the sample chamber $\left(7.0 \times 10^{2} \mathrm{ergs} / \mathrm{mm}^{2} \cdot \mathrm{sec}\right.$ in replication 1 and $1.8 \times 10^{2} \mathrm{ergs} / \mathrm{mm}^{2} \cdot \mathrm{sec}$ in replications 2,3 , and 4 ). The time of photoreactivation treatment was 20 minutes in 
replication 1 and one hour in replications 2,3 , and 4 . The cultures were then kept in the dark at $15^{\circ}-20^{\circ} \mathrm{C}$ for 24 hours until usual incubation under light. To measure the germination rate, more than 500 spores in each culture were scored at the end of five to seven day incubation.

\section{RESULTS AND DISCUSSION}

The u.v.-induced depression of spore germination was reactivated by the postirradiation treatment with visible light at $25^{\circ} \mathrm{C}$, but not at $2^{\circ} \mathrm{C}$ (Table 1 ). This indicates that moss spores are capable of enzymatic photoreactivation of u.v.-induced damage.

Photoreactivation efficiencies at various wavelengths are shown in Fig. 1. The reactivation efficiency is expressed in terms percent photoreactivation $\mathrm{R}$ defined as

$$
R=\frac{G_{L}-G_{D}}{G_{0}-G_{D}} \times 100
$$

where the $G_{L}$ is a germination rate of spores subjected to u.v. and photoreactivation treatments, $G_{D}$ a germination rate of spores irradiated with u.v. and kept in the dark, and $G_{0}$ a germination rate of control spores. As shown in Fig. 1, photoreactivation occurred at 4365 and $4610 \AA$. The former wavelength was more efficient than the latter. None of the other seven wavelengths tested showed any statistically significant reactivation. The observed region of photoreactivation-effective wavelengths is narrower than those in microorganisms mentioned in Introduction.

We have not yet obtained a complete curve for u.v. inactivation of germination in moss spores because each sporogon contains limited number of spores. This is the reason why we used the values of percent photoreactivation $\mathrm{R}$ in Fig. 1.

Photoreactivation kinetics at $4610 \AA$ was obtained by varying the illumination time from 10 to 120 minutes. The germination rate increased initially with time and then reached a saturation at about 60 minutes (Fig. 2). To our surprise, u.v. irradiated spores left near a window for about 6 hours in a daytime at $15^{\circ}$ to $20^{\circ} \mathrm{C}$ showed much higher level of reactivation than the maximal reactivation reached with $4610 \AA$. The nature of this reactivation is unknown.

The spore population used in Fig. 2 seems to have been incidentally of a sensitive type as will be seen from comparing with spores in Table 1. However, the germina-

Table 1. Effect of temperature during the photoreactivation treatment on germination rates of $u . v$. irradiated moss spores

\begin{tabular}{ccccc}
\hline & Control* & U.V.* & \multicolumn{2}{c}{ Photoreactivated at** } \\
& & & $2^{\circ} \mathrm{C}$ & $25^{\circ} \mathrm{C}$ \\
\hline Germination Rate $(\%)$ & 92 & 42 & 45 & 69 \\
\hline
\end{tabular}

* Steeped control spores (non-irradiated) or u.v. irradiated spores $\left(3 \times 10^{4} \mathrm{ergs} / \mathrm{mm}^{2}\right)$ were kept in the dark at $25^{\circ} \mathrm{C}$ for 24 hours and then incubated under light as in an usual manner (see text).

** U.v. irradiated spores were exposed at $2^{\circ} \mathrm{C}$ or $25^{\circ} \mathrm{C}$ to photoreactivating light from a daylight type fluorescent lamp for one hour. After the photoreactivation treatment the spores were kept in the dark at $25^{\circ} \mathrm{C}$ for 20 hours, and then incubated under light (see above). 


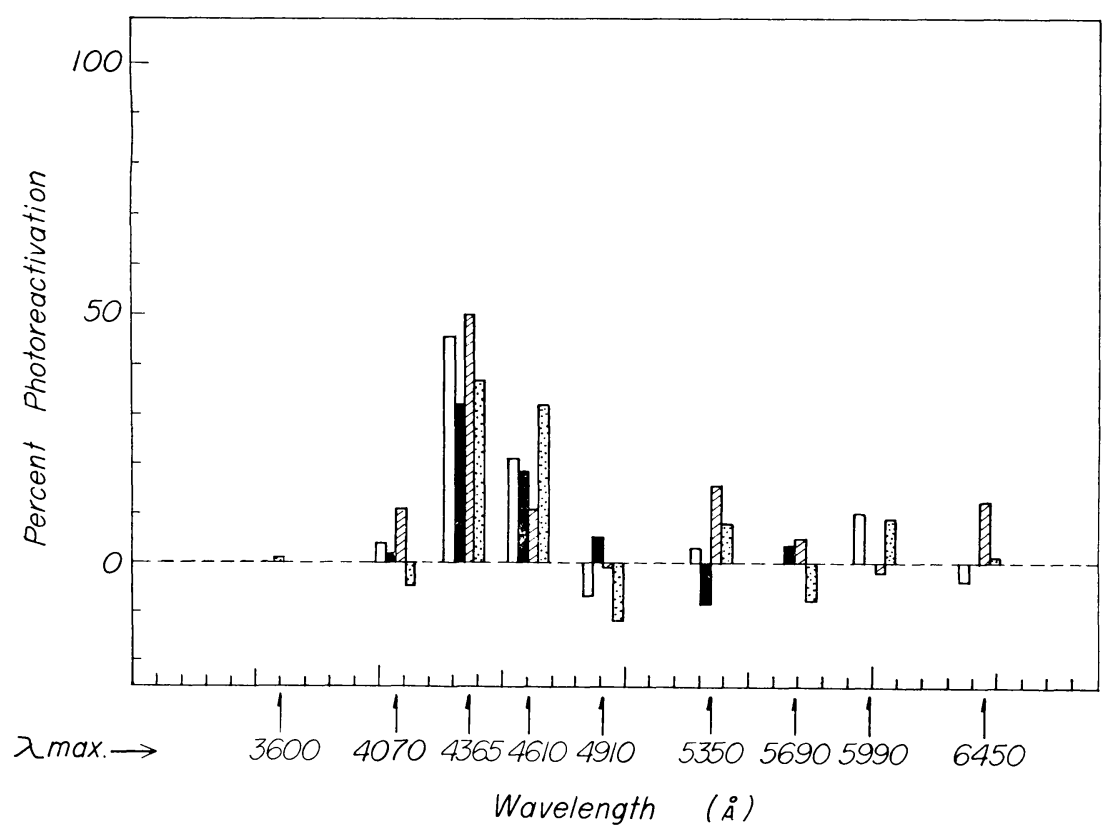

Fig. 1. Percent photoreactivation with monochromatic light of u.v.-induced-inhibition of germination in moss spores. U.v. irradiated spores $\left(3 \times 10^{4} \mathrm{ergs} / \mathrm{mm}^{2}\right)$ were illuminated around $20^{\circ} \mathrm{C}$ with varying wavelength of photoreactivating light $\left(\mathrm{ca} .8 \times 10^{5}\right.$ ergs $/ \mathrm{mm}^{2}$ in replication 1 and ca. $6 \times 10^{5} \mathrm{ergs} / \mathrm{mm}^{2}$ in replications 2,3 and 4). The conditions of post-illumination incubation and of scoring germination rates are the same as described in the footnote of Table 1. Four kinds of columns at each wavelength of photoreactivating light represent four replications of independent experi-

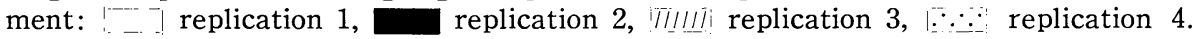
The definition of the percent photoreactivation $\mathrm{R}$ is given in the text.

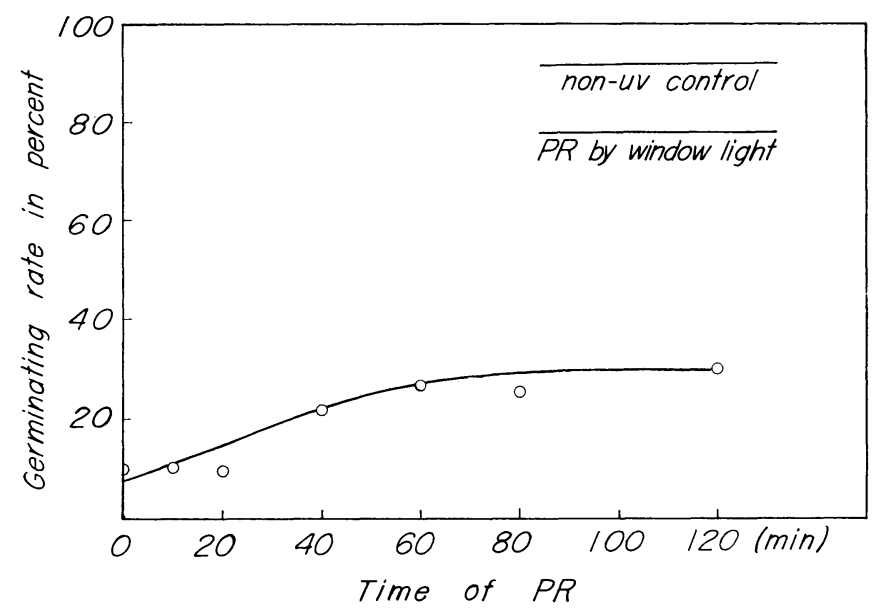

Fig. 2. Photoreactivation kinetics for germination rates of moss spores inactivated by u.v. U. v. irradiated spores at $3 \times 10^{4} \mathrm{ergs} / \mathrm{mm}^{2}$ were exposed to a photoreactivating light at $4610 \AA$ at an intensity of $7 \times 10^{2} \mathrm{ergs} / \mathrm{mm}^{2} \cdot \mathrm{sec}$. After the photoreactivation treatment the spores were kept in the dark for 24 hours, then transferred to an usual incubation condition under light. A marked recovery in germination rate of u.v. irradiated spores after exposure to natural window light is also shown. 
tion rate at no u.v. dose and the photoreactivating ability were normal in this spore population.

Jagger et al. (1970) classified photoreactivation phenomena into three types: Type I is the true enzymatic photoreactivation, Type II is equivalent to photoprotection in its molecular mechanism, and Type III is a new kind of direct photochemical recovery. Type III is said to occur at wavelengths shorter than $4000 \AA$. The unique feature of the present work is the failure of recovery at $4070 \AA$ or shorter (Fig. 1). This and the lack of the recovery by the pre-u.v. irradiation of visible light to spores (unpublished) support the view that the photoreactivation observed in moss spores is of Type I. The cause of the failure of photoreactivation in the near-u.v. region is not clear. It could be the case that near-u.v. does not penetrate into nucleus due to the screen of spore wall and cytoplasmic organelles. The nature of the observed photoreactivation in moss spores would be elucidated if we can make a successful demonstration of photoreactivating enzyme in it in future. The possibility of the third kind of photoreactivation (Type III) is not yet checked.

\section{SUMMARY}

The germination of the spores of Bryum pseudo-triquetrum was much reduced after irradiation with germicidal ultraviolet light. This reduction was photoreactivated by the irradiation with monochromatic light at 4365 and $4610 \AA$ but not at 4070 or $3600 \AA$. At $4610 \AA$, the reactivation rate increased with illuminating time and saturated beyond 60 minutes. The reactivation occurred at $25^{\circ} \mathrm{C}$, but not at $2^{\circ} \mathrm{C}$. These results suggest that the observed photoreactivation is of the enzymatic nature.

\section{ACKNOWLEDGMENTS}

The authors wish to express their sincere thanks to Professor Sohei Kondo, Osaka University, for the critical reading and correction of the original manuscript.

\section{LITERATURE CITED}

Amano, E., and T. Mabuchi, 1966 Photoreactivation of UV-induced damage in maize pollen. Ann. Rep. Natl. Inst. Genet. 17: 114-115.

Dulbecco, R., 1950 Experiments on photoreactivation of bacteriophages inactivated with ultraviolet radiation. J. Bact. 59: 329-347.

Fujii, T., 1969 Photoreactivation of mutation induced by ultraviolet radiation of maize pollen. Rad. Bot. 9: 115-124.

Ikenaga, M., and T. Mabuchi, 1966 Photoreactivation of endosperm mutation by ultraviolet light in maize. Rad. Bot. 6: 165-169.

Jagger, J., 1967 In “Introduction to Research in Ultraviolet Photobiology” pp. 108-118. PrenticeHall, Inc., N. J.

Jagger, J., S. Stafford, and J. M. Snow, 1969 Thymine-dimer and action-spectrum evidence for indirect photoreactivation in Escherichia coli. Photochem. Photobiol. 10: 383-395.

Jagger, J., H. Takebe, and J. M. Snow, 1970 Photoreactivation of killing in Streptomyces: Action spectra and kinetic studies. Photochem. Photobiol. 12: 185-196. 
Kelner, A., 1951 Action spectra for photoreactivation of ultraviolet irradiated E. coli and S. griseus. J. Gen. Physiol. 34: 835-852.

Kinugawa, K., 1966 Some morphological and physiological observations on the sporelings of Bryum pseudo-triquetrum. Bot. Mag. Tokyo. 79: 674-686

Kondo, S., and T. Kato, 1966 Action spectra for photoreactivation of killing and mutation to prototrophy in UV-sensitive strains of Escherichia coli possessing and lacking photoreactivating enzyme. Photochem. Photobiol. 5: 827-837.

Matsumura, S., and T. Mabuchi, 1966 Photoreactivation of an UV-induced mutation in maize. Ann. Rep. Nat1. Inst. Genet. 17: 112-113.

Rupert, C. S., 1960 Photoreactivation of transforming DNA by an enzyme from baker's yeast. J. Gen. Physiol. 43: 573-595.

Saito, N., and H. Werbin, 1969 Evidence for a DNA-photoreactivating enzyme in higher plants. Photochem. Photobiol. 9: 389-393.

Schiff, T., H. Lyman, and H. Epstein, 1961 Studies of chloroplast in Euglena II. Photoreversal of the UV inhibition of green colony formation. Biochim. Biophys. Acta. 50: 310-318.

Setlow, J. K., and N. E. Boiling, 1963 The action spectrum of an in vitro DNA photoreactivation system. Photochem. Photobiol. 2: 471-477.

Setlow, J. K., 1966 The molecular basis of biological effects of ultraviolet radiation and photoreactivation. Current Topics in Radiation Research 2: 195-248.

Van Baalen, C., 1968 The effects of ultraviolet irradiation on a coccoid blue-green alga: survival, photosynthesis, and photoreactivation. Plant Physiol. 43: 1689-1695. 